Forthcoming in Journal of International Economics

\title{
The Purchasing Power Parity Persistence Paradigm
}

\author{
Christian J. Murray and David H. Papell \\ University of Houston
}

June 29, 2000

\begin{abstract}
Rogoff (1996) describes the "remarkable consensus" of 3-5 year half-lives of purchasing power parity deviations among studies using long-horizon data. These studies, however, focus on rejections of unit roots in real exchange rates and do not use appropriate techniques to measure persistence. Our half-life estimates explicitly account for serial correlation, sampling uncertainty and, most importantly, small sample bias. Calculating confidence intervals as well as point estimates for long-horizon and post-1973 data, we find that, even though most of the point estimates lie within the 3-5 year range, univariate methods provide virtually no information regarding the size of the half-lives.
\end{abstract}

Correspondence to:

Department of Economics, University of Houston, Houston, TX 77204-5882

Christian Murray, phone: (713) 743-3835, fax: (713) 743-3798, e-mail: cjmurray@uh.edu David Papell, phone: (713) 743-3807, fax: (713) 743-3798, e-mail: dpapell@uh.edu

We thank Charles Engel, Bruce Hansen, Lutz Kilian, Charles Nelson, James Stock, seminar participants at the Texas Econometrics Camp IV and the University of Houston, and especially two anonymous referees for helpful comments and discussions. 


\section{Introduction}

In a recent but already celebrated article, Rogoff (1996) describes the "purchasing power parity puzzle" as the question of how to reconcile high short-term volatility of real exchange rates with extremely slow convergence to purchasing power parity (PPP). Reviewing the empirical literature, he finds a "remarkable consensus" of 3-5 year half-lives of PPP deviations among studies using long-horizon data, seemingly far too long to be explained by nominal rigidities. Studies using panel methods with post-1973 floating real exchange rates, such as Wu (1996) and Papell (1997), find only slightly shorter (2.5 year) half lives.

There have already been several attempts to "solve" the PPP puzzle. Taylor and Peel (1998) investigate nonlinear mean reversion. Once allowance is made for nonlinearities, the speed of adjustment to real exchange rate shocks may be much greater than what is found with linear models. Hegwood and Papell (1998) consider structural change. Once the effects of occasional permanent disturbances to real exchange rates are accounted for, the half-lives of PPP deviations in long-horizon data are much reduced. Cheung and Lai (2000) use impulse response analysis and compute confidence intervals, as well as point estimates, of half-lives of PPP deviations for several post-1973 real exchange rates. The lower bounds of the confidence intervals are all less than 1.5 years, apparently low enough to be explained by models with nominal rigidities.

The evidence of 3-5 year half lives of PPP deviations in the long-horizon studies cited by Rogoff comes primarily from two sources of data. Abuaf and Jorion (1990) use data collected by Lee (1976) on bilateral, WPI based, real exchange rates between the United States and eight countries for 1900-1972 and find average half-lives of 3.3 years. Glen (1992) and Cheung and Lai (1994) find similar results with the data updated through 1987 and 1992. Frankel (1986) uses 
a 116 year long data set for the WPI based dollar/pound real exchange rate and reports a half-life of 4.6 years. Lothian and Taylor (1996) use two centuries of data for the dollar-pound rate and find an almost identical half-life of 4.7 years. ${ }^{1}$ The evidence of 2.5 year half-lives with post-1973 data in Wu (1996) and Papell (1997) comes from quarterly, CPI based, real exchange rates for industrialized countries with the United States dollar as the numeraire currency.

The papers that attempt to solve the PPP puzzle take the 3-5 year half-life consensus as a starting point for their analysis. The long-horizon evidence that Rogoff cites, however, comes from papers that were primarily concerned with the question of whether unit roots in real exchange rates could be rejected and do not use appropriate techniques to measure persistence. These papers typically conduct Dickey-Fuller (DF) unit root tests, regressing the real exchange rate on a constant and its lagged value. If the coefficient on the lagged real exchange rate is significantly less than one, the unit root null is rejected in favor of the alternative of level stationarity. The half-life, the expected number of years for a PPP deviation to decay by 50 percent, is calculated from the coefficient on the lagged real exchange rate.

We identify three issues involving the use of these half-lives as evidence of the persistence of PPP deviations: confidence intervals, serial correlation and impulse response functions, and small sample bias.

First, simply reporting the point estimates of the half-lives provides an incomplete picture of the speed of convergence towards PPP. Following Cheung and Lai (2000), we supplement the point estimates of $\alpha$ with conventional bootstrap confidence intervals intended to measure the precision of the implied half-life estimates. These confidence intervals, however, are

\footnotetext{
${ }^{1}$ Lothian and Taylor also use the franc-pound rate, and find a half-life of 2.5 years. Diebold, Husted, and Rush (1991), using data for the gold standard period, find average half-lives of 2.8 years.
} 
questionable to the extent that the root of the true process is possibly equal to unity (see Basawa et al. 1991; Hansen 1999; and Inoue and Kilian 2000).

Second, use of the Dickey-Fuller test is only appropriate if the real exchange rate can be well represented as a first order autoregressive processes. If not, Augmented Dickey-Fuller (ADF) tests, which add lagged first differences of the real exchange rate in order to account for serial correlation, must be used. In that case, the half-life calculated from the coefficient on the lagged level of the real exchange rate is no longer an appropriate measure of persistence. For higher order AR models, the half-life must be calculated directly from the impulse response function. Unlike in the $\mathrm{AR}(1)$ model, conventional bootstrap confidence intervals for these halflife estimates are asymptotically valid even in the presence of a possible unit root (see Inoue and Kilian 2000).

Third, and most important, the least squares estimates of the half-lives are biased downward in small samples. This imparts a "double" bias to bootstrap confidence intervals because they are constructed using biased estimates from a biased parameterization. As a result, even if one is confident that PPP holds, so that conventional bootstrap confidence intervals are asymptotically valid, the persistence of PPP deviations can be seriously misrepresented. Moreover, the extent of the bias is greater with more persistent processes. This is especially relevant for real exchange rates, which are often characterized as stationary with highly persistent fluctuations.

Andrews (1993) shows how to calculate exactly median-unbiased estimates of half-lives in DF regressions, as well as exact confidence intervals. Andrews and Chen (1994) show how to perform approximately median-unbiased estimation of AR parameters in ADF regressions. These confidence intervals are asymptotically valid both in the AR(1) case and the AR(p) case. 
Similar corrections based on mean-unbiased estimates of the AR parameters have been proposed by Kilian (1998).

The purpose of this paper is to provide evidence on the half-life of PPP deviations, with a focus on whether Rogoff's 3-5 year consensus can be supported. We utilize two data sets. First, starting with the Lee (1976) data, we construct annual, WPI based, U.S. dollar real exchange rates from 1900-1996 for eight countries. Second, using nominal exchange rates and CPIs for the post Bretton Woods period, we construct quarterly, U.S. dollar real exchange rates from 1973:11998:2 for twenty industrial countries. While we estimate DF and ADF regressions, we are not concerned with the statistical question of whether unit roots in real exchange rates can be rejected and report no test statistics. ${ }^{2}$

In order to provide a benchmark, we start by running DF regressions and calculating bootstrap confidence intervals for the half-lives with long-horizon annual data. While the average half-life is in accord with previous studies, the confidence intervals apparently cast doubt on the 3-5 year consensus. Almost all of the lower bounds are below 2 years, and several of the upper bounds are above 2 years. A similar picture emerges with ADF regressions. Allowing for serial correlation generally lowers the half-lives, while using the impulse response functions raises them, but the differences are not dramatic. However, as discussed above, the AR(1) bootstrap confidence intervals are likely to be theoretically invalid and misleading in practice.

Correcting the bias in the estimates, however, provides a very different perspective. For the DF regressions, the exactly median-unbiased estimates of the half-lives are, as expected, larger than the least squares estimates. The lower bounds of the confidence intervals, while

\footnotetext{
${ }^{2}$ Univariate and panel unit root tests are conducted by Abuaf and Jorion (1990) for long-horizon data and by Papell (1997) for post-1973 data.
} 
larger than the least squares estimates, are still below 2 years for most of the real exchange rates. More importantly, the upper bounds on the confidence intervals are extremely large, over 20 years in five of the six cases. It is worth emphasizing that the least squares point estimates of half-lives from DF regressions provide the evidence that is the basis of Rogoff's 3-5 year consensus. Once we correct the bias and calculate confidence intervals, however, we cannot even claim to have evidence that the half-lives fall within a 2-20 year range. Incorporating serial correlation by estimating ADF regressions and calculating half-lives from the impulse responses tightens the confidence intervals, but most of the upper bounds are still well above 5 years.

We proceed to perform the same analysis for post-1973 quarterly real exchange rates. This data has two advantages over the long-horizon data; it does not mix observations from different nominal exchange rate regimes and data is available for more countries, and one disadvantage; the time span of the data is much shorter.

Cheung and Lai (2000), using monthly data on four real exchange rates over a similar period, calculate confidence intervals based on impulse response functions for least squares estimates of half-lives of PPP deviations from ARMA models. The lower bound of their confidence intervals are all below 1.5 years, leading them to conclude that, since this half-life is short enough to be explained by models with nominal rigidities, there is no PPP puzzle. Using quarterly data, we confirm that their result generalizes to more currencies. Whether we compute DF regressions, ADF regressions with the half-life calculated from the point estimate of the lagged real exchange rate, or $\mathrm{ADF}$ regressions with the half-life calculated from the impulse response function, the lower bounds of the confidence intervals are below 1.5 years in almost every case. Moreover, most of the upper bounds are below 5 years. 
This "solution" of the PPP puzzle is problematic for two reasons. First, as discussed previously, the DF results are unlikely to be valid theoretically. Second, the result that the confidence intervals fall within a narrow range does not survive the use of median-unbiased estimates. For the DF regressions, while most of the lower bounds of the confidence intervals remain under 1.5 years, every upper bound is infinite. The picture does not change much with the ADF regressions. When the half-lives are calculated from the impulse response functions, all but one of the lower bounds are below 1.5 years. But nineteen of the twenty upper bounds are above 10 years, with sixteen of the twenty infinite. These confidence intervals are so wide that they provide absolutely no information regarding the speed of convergence of PPP deviations.

\section{Persistence with Annual Long-Horizon Data}

Most of the evidence of 3-5 year half-lives of PPP deviations in long-horizon data, including the papers of Abuaf and Jorion (1990) and Glen (1992) surveyed by Rogoff (1996), comes from the data collected by Lee (1976). We use Lee's nominal exchange rate and WPI data, updated through 1996 with data from the International Financial Statistics CD-ROM, to construct U.S. dollar based, annual real exchange rates for six countries: Canada, France, Italy, Japan, Netherlands, and the UK. ${ }^{3}$

\subsection{Least Squares Estimates}

Estimates of Dickey-Fuller regressions, which replicate (with extended samples) the results of the studies that provide the basis of the 3-5 year half-life consensus, are provided in Table 1. The DF regression takes the form,

$$
q_{t}=c+\alpha q_{t-1}+u_{t}
$$

\footnotetext{
${ }^{3}$ Abuaf and Jorion (1990) also use data for Germany, which contains gaps, and for Switzerland, which does not start until 1912.
} 
where $\mathrm{q}_{\mathrm{t}}$ is the dollar denominated real exchange rate. The half-life in equation (1), defined as the number of periods required for a unit shock to dissipate by one half, is calculated as $\ln (0.5) / \ln (\alpha)$. The least squares estimates of $\alpha$ range from 0.595 (France) to 0.889 (Japan), implying point estimates of the half-life of PPP deviations from 1.34 years (France) to 6.51 years (Japan). The average half-life is 3.57 years and the median half-life is 3.01 years. Abuaf and Jorion (1990), with similar data through 1972, found an average half-life of 3.3 years. It is interesting that adding 24 years of post- 1973 flexible exchange rate data to their sample makes so little difference to the results.

The point estimates of the half-lives are on the low side of Rogoff's 3-5 year consensus. That figure, however, is skewed upward because, for reasons of data availability, a number of the long-horizon PPP studies use the pound/dollar exchange rate. The half-life for the pound/dollar rate, 4.86 years, is the second longest among the six countries. Since Rogoff's consensus is based on several studies using Lee data and several using pound/dollar data, the relatively slowly mean reverting pound/dollar rate receives a disproportionate weight. If the "consensus" is widened slightly, to 2.5 - 5 years, the half-lives of four of the six real exchange rates fall within the range.

As in Cheung and Lai (2000), we report 95\% bootstrap confidence intervals for the halflives of the PPP deviations in Table 1, so that we may assess the sampling variability of the least squares estimates. We use a parametric percentile bootstrap. For each parameterization in Table 1, we generate 5000 artificial time series with iid Normal errors, and construct a confidence interval for the least squares half-life. The average lower bound of the confidence intervals is 
1.42 years and the average upper bound is 6.15 years, while the median lower bound is 1.33 years and the median upper bound is 5.02 years. $^{4}$

If $\alpha<1$ in Equation (1), the bootstrap confidence interval is valid asymptotically. However, if $\mathrm{c}=0$ and $\alpha=1$, so that the real exchange rate follows a driftless random walk (the null hypothesis in DF tests for PPP), Basawa et al. (1991) prove that the bootstrap is asymptotically invalid. Moreover, its small-sample properties will be poor to the extent that the root of the true process is close to unity; see Kilian (1999). Finally, Equation (1) ignores the presence of serial correlation in real exchange rate data. For these reasons, we suspect the accuracy of the confidence intervals in Table 1.

Least squares estimates of Augmented Dickey-Fuller regressions that account for serial correlation are presented in Table 2. The ADF regression takes the form,

$$
q_{t}=c+\alpha q_{t-1}+\sum_{i=1}^{k} \psi_{i} \Delta q_{t-i}+u_{t}
$$

We use the general-to-specific lag selection procedure studied by Hall (1994) and Ng and Perron (1995), with the maximum lag set to $8 .^{5}$ With the exception of Italy, at least one lag of the first differences of the real exchange rates in Equation (2) is significant, and so the ADF half-lives differ from the DF half-lives for the other five countries. When the half-lives are calculated from the value of $\alpha$, they fall for four of the five countries, but nearly double for Japan. The median point estimate of the half-life is 2.53 years, with a median lower bound of 1.17 years and a median upper bound of 4.19 years. ${ }^{6}$ These estimates are somewhat lower than the estimates from the DF regressions.

\footnotetext{
${ }^{4}$ Further research is needed to assess the sensitivity of our results with respect to departures from Normality.

${ }^{5}$ We use $10 \%$ as the criterion for significance.

${ }^{6} \mathrm{We}$ focus on the median values to mitigate the effect of the one outlier.
} 
The half-life calculated from the value of $\alpha$ assumes that shocks to real exchange rates decay monotonically. While appropriate for a DF regression based on an $\mathrm{AR}(1)$, this assumption is inappropriate for ADF regressions, since in general shocks to an $\mathrm{AR}(\mathrm{k}+1)$ will not decay at a constant rate. In light of this we calculate the half-life from on the impulse response function of an $\mathrm{AR}(\mathrm{k}+1)$. The half-life is defined as the number of years required for a unit impulse to dissipate by one half. ${ }^{7}$

Half-lives based on the impulse response functions are presented in Table 2. All of the point estimates of the half-lives (except for Italy) increase, and the half-life for Japan becomes infinite. The median point estimate of the half-lives is 3.39 years, with a median lower bound of 1.52 years and a median upper bound of 4.84 years.

In contrast to the $\mathrm{AR}(1)$ model with zero intercept, the bootstrap is not necessarily asymptotically invalid for higher order AR models if there is a unit root present. Inoue and Kilian (2000) prove that if $c=0$ and $\alpha=1$ in higher order AR models, the bootstrap will be asymptotically invalid for $\alpha$, but asymptotically valid for the individual slope parameters in the AR representation of Equation (2):

$$
q_{t}=c+\sum_{i=1}^{k+1} \phi_{i} q_{t-i}+u_{t}
$$

where $\phi_{1}=\alpha+\psi_{1}, \phi_{\mathrm{j}}=\psi_{\mathrm{j}}-\psi_{\mathrm{j}-1}$ for $\mathrm{j}=2, \ldots, \mathrm{k}$, and $\phi_{\mathrm{k}+1}=-\psi_{\mathrm{k}}$. Thus, the half-lives in Table 2 based on $\alpha$ will be invalid asymptotically if there is a unit root, but the half-lives based on the impulse response function (based on $\phi_{1}, \ldots, \phi_{\mathrm{k}+1}$ ) will be asymptotically valid.

\footnotetext{
${ }^{7}$ For DF regressions, half-lives calculated from impulse response functions are the same as half-lives calculated from the value of $\alpha$.
} 
We have estimated half-lives of PPP deviations from three specifications. The median point estimates are between 2.5 and 3.5 years, the median lower bounds of the confidence intervals are between 1 and 1.5 years, and the median upper bounds do not exceed 5 years. It is tempting to conclude that the half-lives of PPP deviations are somewhat lower than Rogoff's 3-5 year consensus, and possibly low enough to be explained by models with nominal rigidities. There are two reasons why this conclusion would be erroneous. First, as discussed earlier, the bootstrap confidence intervals for the half-lives based on $\alpha$ will be asymptotically invalid if $c=0$ and $\alpha=1$. Second, there are serious finite sample problems if the true value of $\alpha$ is close to or equal to unity. The least squares estimator of $\alpha$, and thus of the half-lives of PPP deviations, is biased downward, with the bias becoming larger the closer the true $\alpha$ is to unity. ${ }^{8}$ This is not an issue in testing for unit roots, as it is accounted for in calculating the critical values, but is clearly an issue in measuring persistence. While this small sample bias applies to both the point estimates and the confidence intervals, it is more severe for the confidence intervals. This is because we are generating artificial data based on a biased parameterization, and then obtaining biased estimates from this parameterization. The severity of this added layer of bias can be such that the conventional bootstrap interval does not contain the least squares estimate. ${ }^{9}$

\subsection{Median-unbiased Estimates}

We correct for the bias in least squares estimates by calculating median-unbiased point estimates and confidence intervals for $\alpha$ in Dickey-Fuller and Augmented Dickey-Fuller regressions. Median-unbiased estimators of scalar parameters have the desirable properties that

\footnotetext{
${ }^{8}$ While the bias is always downward in the AR(1) model, it may not be in higher order models with low persistence. See Stine and Shaman (1989) for details. The real exchange rates that we study are all sufficiently persistent so that the potential bias reversal is not relevant.

${ }^{9}$ Kilian (1998) discusses this issue.
} 
their point estimates remain median-unbiased and the coverage probabilities of their confidence intervals are invariant under monotonic transformations (see Andrews and Phillips, 1987). For example, in Table 1 we are taking a monotonic transformation of $\alpha_{L S}, \ln (0.5) / \ln \left(\alpha_{L S}\right)$ to obtain the least squares estimate of the half-life. If we replace $\alpha_{\mathrm{LS}}$ with a median-unbiased estimate, $\alpha_{\mathrm{MU}}$, the half-life estimate will also be median-unbiased.

We use the technique of Andrews (1993) to calculate exactly median-unbiased estimates of $\alpha$ in equation (1). This allows us to calculate exactly median-unbiased point estimates, as well as exact confidence intervals, of the half-lives of PPP deviations. Let $\alpha_{L S}$ denote the least squares estimate of $\alpha$ in equation (1). To calculate the median-unbiased estimate, we find the value of $\alpha$ that yields the least squares estimator to have a median of $\alpha_{\mathrm{LS}}$. For example, Andrews (1993) demonstrates that if the true $\alpha$ is 0.90 and $\mathrm{T}=100$, then the median of $\alpha_{\mathrm{LS}}$ in Equation (1) is 0.869; least squares has a median bias of -0.031 . Thus, if $\alpha_{L S}=0.869$, our median-unbiased estimate of $\alpha$ is 0.90 . In general, analytic forms for median-unbiased estimators do not exist, and simulation techniques are thus necessary. ${ }^{10}$

Another technique for correcting the bias of least squares estimators is mean-unbiased estimation. Mean-unbiasedness is what is traditionally implied when an estimator is said to be unbiased; that is, the expected value of an estimator is equal to the true parameter value. ${ }^{11}$ For calculating half-lives in the $\mathrm{AR}(1)$ model, we prefer median-unbiased estimation to meanunbiased estimation because the latter will not be unbiased under the half-life transformation in the $\mathrm{AR}(1)$ model. For the $\mathrm{AR}(\mathrm{p})$ model, both mean and median-unbiasedness are ad hoc and

\footnotetext{
${ }^{10}$ A drawback to the median-unbiased estimators of $\alpha$ in Equations (1) and (2) is that they do have an explicit optimality property; we do not know whether they are the best (minimum variance) median-unbiased estimators.

${ }^{11}$ See Shaman and Stine (1988) and Shaman and Stine (1989) for mean-unbiased estimation of AR models.
} 
there is no compelling reason to prefer one method to the other, although both methods appear to be successful at reducing the bias of the impulse response estimates (see Andrews and Chen 1994; and Kilian 1998, 1999). Here we use median-unbiased methods throughout for internal consistency. Further research is needed to assess the sensitivity of our results to other methods of bias correction.

Exactly median-unbiased estimates of the half-lives of PPP deviations from DickeyFuller regressions are presented in Table $3 .^{12}$ Compared with Table 1, which reports least squares estimates of the same regressions, all of the point estimates of the half-lives increase. The average half-life rises from 3.57 years to 4.89 years, while the median half-life rises from 3.01 years to 3.49 years. Although higher than the least squares estimates, the median-unbiased point estimates are consistent with the 3-5 year consensus for PPP deviations.

The $95 \%$ confidence intervals of exactly median-unbiased estimates of half-lives of PPP deviations from the DF regressions are also presented in Table 3. The lower bounds of the confidence intervals are not much higher than those derived from the least squares estimates, with the median lower bound increasing from 1.33 years to 1.77 years. The upper bounds of the confidence intervals, however, change dramatically. The median upper bound is 28.54 years, compared with the least squares median upper bound of 5.02 years. All of the upper bounds, except for France, are above 20 years, and two are infinite. With 100 years of data, such wide confidence intervals provide almost no information on the half-lives of PPP deviations.

Why are the confidence intervals for median-unbiased half-lives so much wider than their least squares counterparts? Compare the least squares confidence intervals for $\alpha$ in Table 1 to

\footnotetext{
12 These estimates are computed by constructing Andrews' (1993) Table 2 for T=97, with $\alpha$ ranging from -0.99 to 1.0 , in increments of 0.01 . The distribution of $\alpha$ does not depend on the constant term, which we set to zero in the simulation.
} 
the median-unbiased confidence intervals in Table 3. The least squares interval is wider than the median-unbiased interval in four of the six cases. However, the median-unbiased half-life intervals are much wider than the least squares half-life intervals for all six exchange rates. This is because the median-unbiased intervals for $\alpha$ lie closer to unity, and were are taking a convex transformation to obtain the half-life intervals. For example, compare the intervals $[0.80,0.90]$ and $[0.85,0.95]$, each with width of 0.1 . If we take the half-life transformation of each lower and upper bound, we end up with $[3.11,6.58]$ and $[4.27,13.51]$. The width of the former is 3.47 while the width of the latter is 9.24 . It is the transformation that is driving the greater width of medianunbiased half-life intervals, not a higher variance of $\alpha_{\mathrm{MU}}$ relative to $\alpha_{\mathrm{LS}}$.

As discussed in Andrews and Chen (1994), in AR(k+1) models with $\mathrm{k}>0$, the medianunbiased estimator of $\alpha$ is no longer exact, but approximate. This is because the medianunbiased estimator of $\alpha$ depends on the true values of the $\psi_{\mathrm{i}}$ terms in Equation (2), which are unknown. Andrews and Chen (1994) propose a computationally intensive iterative procedure to obtain approximately median-unbiased estimates of $\alpha, \psi_{1}, \ldots, \psi_{\mathrm{k}}$, as well as impulse responses and cumulative responses. ${ }^{13}$ The intuition behind obtaining the approximately median-unbiased estimate of $\alpha$ in equation (2), $\alpha_{\mathrm{AmU}}$, is the same as in the exactly median-unbiased case. Conditional on the least squares estimates of $\psi_{1}, \ldots, \psi_{\mathrm{k}}$, we find the value of $\alpha$ which yields the least squares estimator to have $\alpha_{\mathrm{LS}}$ as its median; call this $\alpha_{1, \mathrm{AMU}}$. Conditional on $\alpha_{1, \mathrm{AMU}}$, we obtain a new set of estimates of $\psi_{1}, \ldots, \psi_{\mathrm{k}}$ and proceed to calculate a new median-unbiased estimate of $\alpha$ conditional on these coefficients; call this $\alpha_{2, \mathrm{AMU}}$. $\alpha_{\mathrm{AMU}}$ is obtained when convergence occurs.

\footnotetext{
${ }^{13}$ Stock (1991) calculates asymptotically median-unbiased estimates of the largest AR root, which is in general different from $\alpha$.
} 
Andrews and Chen (1994) report simulation evidence demonstrating that the approximation is quite accurate; that is $\alpha_{\mathrm{AMU}}, \psi_{1, \mathrm{AMU}}, \ldots, \psi_{\mathrm{k}, \mathrm{AMU}}$ are essentially median-unbiased. For AR(2) models, the impulse responses are essentially median-unbiased for all lags. For higher order AR models the impulse response estimates are downward median-biased, but the downward bias is much less than the least squares estimates.

Point estimates and $95 \%$ confidence intervals of approximately median-unbiased estimates of half-lives of PPP deviations from ADF regressions are presented in Table 4. Since these regressions allow for (but do not impose) serial correlation and correct for bias, they are the preferred specification. When the half-lives are calculated from the value of $\alpha$, they fall for four of the six countries, are the same for Italy (because $\mathrm{k}=0$ ), and become infinite for Japan. This is the same pattern as was observed for the least squares estimates. The median point estimate of the half-life is 2.95 years, with a median lower bound of 1.49 years and a median upper bound of 12.17 years. $^{14}$ While the confidence intervals of approximately median-unbiased estimates of half-lives of PPP deviations from ADF regressions are narrower than from DF regressions, they are still too wide to be informative.

Half-lives of approximately median-unbiased estimates of PPP deviations calculated from the impulse response functions are also presented in Table 4. All of the point estimates of the half-lives (except for Italy where $\mathrm{k}=0$ and for Japan which was already infinite) increase. The median point estimate of the half-life is 3.98 years, with a median lower bound of the confidence interval of 1.69 years and a median upper bound of 12.75 years. ${ }^{15}$ These confidence intervals are similar to the confidence intervals when the half-lives are calculated from the value of $\alpha$.

\footnotetext{
14 In this case, the dispersion of the upper bounds is very wide, with the bottom three between 1.67 and 7.35 years, and the top three between 16.98 years and infinity.

15 The dispersion of the upper bounds is also very wide in this case.
} 
We have calculated median-unbiased estimates of PPP deviations for three specifications: DF regressions, ADF regressions with half-lives calculated from the value of $\alpha$, and ADF regressions with half-lives calculated from the impulse response functions. For all three specifications, the median values of the half-lives are (at least approximately) within the 3-5 year range of Rogoff's consensus. The confidence intervals, however, are so wide as to render the point estimates almost completely uninformative.

\section{Persistence with Quarterly Post-1973 Data}

The second data set that we analyze consists of quarterly, CPI based, real exchange rates from 1973:1-1998:2 for twenty industrialized countries with the United States dollar as the numeraire currency. ${ }^{16}$ The half-lives of PPP deviations using quarterly post-1973 real exchange rates appear to be shorter than the 3-5 year consensus from long-horizon data. For example, Papell (1997) finds half-lives of PPP deviations of 1.74 years with univariate ADF regressions and 2.42 years with panel ADF regressions. ${ }^{17}$

Least squares point estimates and $95 \%$ bootstrap confidence intervals for half-lives of PPP deviations from Dickey-Fuller regressions are reported in Table 5. The median half-life is 2.52 years, the median lower bound of the confidence interval is 0.64 years, and the median upper bound is 4.95 years.

Serial correlation would seem to be of obvious importance with quarterly data, and we report least squares estimates for ADF regressions in Table 6. At least three lags were chosen for

\footnotetext{
${ }^{16}$ The data is from International Financial Statistics. Twenty-three countries are considered industrialized by the IMF. We do not use data for Iceland because of the existence of gaps in its CPI and for Luxembourg because it has a currency union with Belgium. The twenty-one remaining countries provide twenty real exchange rates.

${ }^{17} \mathrm{Wu}$ (1996) reports similar results. Frankel and Rose (1996), using post-1973 annual data for industrialized countries, find a half-life of 3.49 years with a panel DF regression. We intend to use panel methods to further investigate some of the issues raised in this paper in subsequent research.
} 
each country, and so the ADF estimates differ from the DF estimates. When the half-lives are calculated from the value of $\alpha$, the median half-life is 1.77 years, the median lower bound is 0.64 years, and the median upper bound is 3.12 years. When the half-lives are calculated from the impulse response function, the median half-life is 2.15 years, the median lower bound is 1.14 years, and the median upper bound is 4.04 years. As with the long-horizon data, estimates of the half-lives are lowered by incorporating serial correlation and raised by being calculated from the impulse response function.

Cheung and Lai (2000), using monthly data from 1973:4 to 1996:12 on four US dollar real exchange rates: the French franc, German mark, Italian lira, and British pound, calculate confidence intervals based on impulse response functions for least squares estimates of half-lives of PPP deviations from ARMA models. While the median of the $95 \%$ lower bounds of their confidence intervals, 1.22 years, is comparable with our results, the median of their point estimates, 3.39 years, and of their upper bounds, 8.05 years, are considerably higher. They also calculate half-lives after the initial shock amplification. In that case, the median of their point estimates, 2.21 years, becomes comparable with our results, the median of the lower bounds on their confidence intervals, 0.79 years, is smaller than ours, and the median of their upper bounds, 5.34 years, is still larger than ours.

Even though the lower bounds of the least squares half-life confidence intervals in Tables 5 and 6 (and those in Cheung and Lai 2000) are less than 2 years, the PPP puzzle is not solved. The bootstrap confidence interval for the half-life based on $\alpha$ will be asymptotically invalid if the data are integrated and non-trending, and the least squares estimates of the half-life are biased downward in small samples. 
Exactly median-unbiased point estimates and confidence intervals for half-lives of PPP deviations in DF regressions are presented in Table 7. Correcting for the bias substantially raises the point estimates. The half-lives for the real exchange rates of four countries: Canada, Japan, Portugal, and Spain, are infinite and the median half-life, 5.69 years, more than doubles from the least squares estimates. The median lower bound for the confidence intervals, 1.43 years, also increases. Even more dramatically, the upper bound for each of the confidence intervals becomes infinite. These confidence intervals are obviously too wide to convey any information.

Andrews (1993), using monthly data from 1973:1 to 1988:7 for six US dollar real exchange rates: the French franc, German mark, Japanese yen, Canadian dollar, Dutch guilder, and British pound, calculates least squares point estimates and exactly median-unbiased point estimates and 90\% confidence intervals for half-lives of PPP deviations in DF regressions. The median half-life for the least squares estimates is 3.18 years. Correcting for the bias, the halflives for the exactly median-unbiased estimates are all (except for the UK) infinite. The median of the lower bounds of the confidence intervals is 1.90 years and the median of the upper bounds is infinite.

On a country-by-country basis, there are some interesting comparisons between our exactly median-unbiased point estimates of half-lives of PPP deviations and Andrews' estimates. For two countries: Canada and Japan, they are both infinite. For France and Germany, our halflife estimates are 5.69 years while, for the Netherlands, it is 4.24 years. Andrews finds infinite half-lives for all three countries. For the UK, our estimate is 4.24 years and Andrews' estimate is 11.5 years. While both studies start at the same time and use the same nominal exchange rate and CPI data to calculate real exchange rates, we use quarterly data for 25 years while Andrews 
uses monthly data for 15 years. We suspect it is the span of the data, not the frequency of observation, which accounts for the differences in the results for the point estimates.

In contrast, the exactly median-unbiased estimates of the upper and lower bounds of the confidence intervals of the half-lives are very similar between the two studies. First, all of the upper bounds are infinite. Second, while the median of our lower bounds for either all twenty countries, 1.43 years, or for the six countries in common, 1.36 years, is smaller than Andrews' median lower bound, 1.90 years, we calculate 95\% confidence intervals while Andrews calculates $90 \%$ confidence intervals. The overall message from the two studies, however, is the same. Once the bias in least squares estimates is corrected by using exactly median-unbiased estimation techniques, the confidence intervals of half-lives of PPP deviations are too wide to place any credence in the point estimates.

Since serial correlation is important in quarterly data, we calculate approximately medianunbiased point estimates and confidence intervals for half-lives of PPP deviations in ADF regressions, and report the results in Table 8 . As with both the long-horizon data and the least squares estimates of DF regressions, accounting for serial correlation lowers persistence and calculating half-lives from the impulse response function raises persistence. When the half-lives are calculated from the value of $\alpha$, the median half-life is 2.39 years, the median lower bound is 0.74 years, and the median upper bound is infinite. When the half-lives are calculated from the impulse response function, the median half-life is 3.07 years, the median lower bound is 1.24 years, and the median upper bound is infinite. ${ }^{18}$

When we considered real exchange rates in long-horizon data, we concluded that

\footnotetext{
${ }^{18}$ We note that in higher order AR models, $\alpha=1$ does not necessarily imply an infinite half-life.
} 
confidence intervals from approximately median-unbiased estimation of PPP half-lives in univariate regressions were too wide to be informative. The results are even starker here. Whether the half-lives are calculated from the values of $\alpha$ or from the impulse response functions, the median lower bounds of the $95 \%$ confidence intervals are below 1.5 years and the median upper bounds are infinite. Once the bias in least squares estimates of univariate ADF regressions is corrected, they provide virtually no information on how long, if ever, it takes for PPP deviations to dissipate. ${ }^{19}$

\section{Conclusions}

What is the persistence of deviations from purchasing power parity? The consensus of empirical research, which we call the "purchasing power parity persistence paradigm," is that the half-lives of PPP deviations are between 3-5 years in studies using univariate methods on annual long-horizon data and are only slightly shorter in studies using quarterly post-1973 data. This consensus, seemingly too long to be explained by models with nominal rigidities, is a key component of Rogoff's (1996) “purchasing power parity puzzle.”

The evidence on half-lives of PPP deviations comes from work whose major concern is the statistical question of whether unit roots in real exchange rates can be rejected. These techniques are not appropriate for measuring persistence for three reasons. First, virtually all existent studies, whether univariate or panel, use least squares estimates which are biased downwards. Following Andrews (1993), we use median-unbiased estimation techniques to correct for the resultant bias in computing half-lives. Second, almost all studies simply report point estimates and, when bootstrap confidence intervals are computed, the methods chosen tend

\footnotetext{
${ }^{19}$ These results are similar to those reported in Kilian and Zha (2000). Within a Bayesian framework, they find that the posterior error bands of half-lives of post Bretton Woods data are much wider that Rogoff's 3-5 year range.
} 
to be unreliable or even invalid. Third, most of the studies either do not account for serial correlation or, if they incorporate serial correlation, do not calculate half-lives from the impulse response functions.

We utilize two data sets. The long-horizon data are annual, WPI based, U.S. dollar real exchange rates for 1900-1996 for six countries. The short-horizon data are quarterly, CPI based, U.S. dollar real exchange rates for 1973:1-1998:2 for twenty countries. The long-horizon data span several nominal exchange rate regimes, while the short-horizon data are for the post-1973 floating rate regime. For each data set, we calculate point estimates and $95 \%$ confidence intervals of half-lives of PPP deviations using least squares and median-unbiased estimation techniques with and without serial correlation.

We focus on the preferred specification: approximately median-unbiased estimates of Augmented Dickey-Fuller regressions that allow for, but do not impose, serial correlation. With long-horizon data, the median value of the point estimates of PPP deviations from the univariate regressions is 3.98 years, almost in the middle of Rogoff's 3-5 year range. The median bounds of the confidence intervals, however, are too wide to be informative. The lower bound, 1.69 years, is small enough to be consistent with models based on nominal rigidities, while the upper bound, 12.75 years, is almost three times the value of the half-life produced by a speed of convergence, 15 percent per year, which Rogoff describes as "glacial".

The evidence from univariate regressions with post-1973 data is similar. With approximately median-unbiased estimates of $\mathrm{ADF}$ regressions, the median value of the point estimates of PPP deviations is 3.07 years, near the bottom, but still within, Rogoff's consensus. The median bounds of the confidence intervals, 1.24 years and infinity, are too wide to contain any information. The lower bound is below the lower bound with long-horizon data, while the 
upper bound is consistent with a unit root in real exchange rates and consequently no convergence to PPP.

Rogoff's "remarkable consensus" of 3-5 year half-lives of PPP deviations was based on the results of studies that did not incorporate serial correlation, did not report confidence intervals, and, most importantly, reported biased estimates. Using median-unbiased estimates that allow for serial correlation, the point estimates of the half-lives of PPP deviations from regressions for both annual long-horizon and quarterly post-1973 data are consistent with Rogoff's consensus. The confidence intervals, however, are so wide that they are consistent with virtually anything from models with nominal rigidities to models where PPP does not hold. The 3-5 year range of point estimates is sometimes interpreted as an "implicit" confidence interval for the estimates. Our calculation of much wider confidence intervals for median-unbiased estimates shows that this interpretation is unwarranted. 


\section{References}

Abuaf, N. and P. Jorion, 1990, Purchasing power parity in the long run, Journal of Finance 45, 157-174.

Andrews, D.W.K., 1993, Exactly median unbiased estimation of first order autoregressive/unit root models, Econometrica 61, 139-165.

Andrews, D.W.K., and H.-Y. Chen, 1994, Approximately median-unbiased estimation of autoregressive models, Journal of Business and Economic Statistics 12, 187204.

Andrews, D.W.K., and P.C.B. Phillips, 1987, Best median-unbiased estimation in linear regression with bounded asymmetric loss functions, Journal of the American Statistical Association 82, 886-893.

Basawa, I.V., A.K. Mallik, W.P. McCormick, J.H. Reeves, and R.L. Taylor, 1991, Bootstrapping unstable first-order autoregressive processes, Annals of Statistics 19, 1098-1101.

Cheung, Y.-W. and K.S. Lai, 1994, Mean reversion in real exchange rates, Economics Letters 46, 251-56.

Cheung, Y.-W. and K.S. Lai, 2000, On the purchasing power parity puzzle, forthcoming, Journal of International Economics.

Diebold, F., Husted, S., and Rush, M. 1991, Real exchange rates under the gold standard, Journal of Political Economy 99, 1252-1271.

Frankel, J., 1986, International capital mobility and crowding out in the U.S. economy: Imperfect integration of financial markets or of goods markets? in: R. Hafer, ed., How open is the U.S. economy?, (Lexington Books, Lexington) 33-67.

Frankel, J. and A. Rose, 1996, A panel project on purchasing power parity: Mean reversion within and between countries, Journal of International Economics 40, 209-224.

Glen, J.H., 1992, Real exchange rates in the short, medium, and long run, Journal of International Economics 33, 147-66.

Hall, A., 1994, Testing for a unit root in time series with pretest data-based model selection, Journal of Business and Economic Statistics 12, 461-70.

Hansen, B., 1999, The grid bootstrap and the autoregressive model, The Review of Economics and Statistics 81, 594-607. 
Hegwood, N. and D. Papell, 1998, Quasi purchasing power parity, International Journal of Finance and Economics 3, 279-289.

Inoue, A, and L. Kilian, 2000, Bootstrapping autoregressive processes with possible unit roots, manuscript, Department of Economics, University of Michigan.

Kilian, L., 1998, Small-sample confidence intervals for impulse response functions, The Review of Economics and Statistics 80, 218-230.

Kilian, L., 1999, Finite-sample properties of percentile and percentile- $t$ bootstrap confidence intervals for impulse responses, The Review of Economics and Statistics 81, 652-660.

Kilian, L., and T. Zha, 2000, Quantifying the uncertainty about the half-life of deviations from PPP: The role of economic priors, manuscript, Department of Economics, University of Michigan.

Lee, M.H., 1976, Purchasing power parity, (Marcel Dekker, New York).

Lothian, J., and M. Taylor, 1996, Real exchange rate behavior: The recent float from the perspective of the past two centuries, Journal of Political Economy 104, 488-509.

Ng, S. and P. Perron, 1995, Unit root tests in ARMA models with data dependent methods for the selection of the truncation lag, Journal of the American Statistical Association 90, 268-281.

Papell, D., 1997, Searching for stationarity: Purchasing power parity under the current float, Journal of International Economics 43, 313-332.

Papell, D. and H. Theodoridis, 1998, Increasing evidence of purchasing power parity over the current float, Journal of International Money and Finance 17, 41-50.

Rogoff, K., 1996, The purchasing power parity puzzle, Journal of Economic Literature 34, 647-668.

Shaman, P. and R.A. Stine, 1988, The bias of autoregressive coefficient estimators, Journal of the American Statistical Association 83, 842-848.

Stine, R.A., and P. Shaman, 1989, A fixed point characterization for bias of autoregressive estimators, Annals of Statistics 17, 1275-1284.

Stock, J., 1991, Confidence intervals for the largest autoregressive root in U.S. macroeconomic time series, Journal of Monetary Economics 28, 435-459. 
Taylor, M. and D. Peel, 1998, Nonlinear mean-reversion in real exchange rates: towards a solution to the purchasing power parity puzzles, manuscript, University of Oxford.

Wu, Y., 1996, Are real exchange rates nonstationary? Evidence from a panel-data test, Journal of Money, Credit and Banking 28, 54-63. 
Table 1. OLS Half-Lives in Dickey-Fuller Regressions Annual Data: 1900-1996

$$
q_{t}=c+\alpha q_{t-1}+u_{t}
$$

\begin{tabular}{|lcccc|}
\hline Country & $\alpha_{L S}$ & $95 \%$ CI & $H L_{L S}$ & $95 \%$ CI \\
\hline Canada & 0.795 & {$[0.596,0.870]$} & 3.02 & {$[1.34,4.98]$} \\
France & 0.595 & {$[0.376,0.717]$} & 1.34 & {$[0.71,2.08]$} \\
Italy & 0.771 & {$[0.575,0.855]$} & 2.67 & {$[1.25,4.42]$} \\
Japan & 0.889 & {$[0.718,0.943]$} & 6.51 & {$[2.09,11.81]$} \\
Netherlands & 0.793 & {$[0.588,0.872]$} & 2.99 & {$[1.31,5.06]$} \\
UK & 0.867 & {$[0.685,0.922]$} & 4.86 & {$[1.83,8.54]$} \\
\hline
\end{tabular}


Table 2. OLS Half-Lives in Augmented Dickey-Fuller Regressions Annual Data: 1900-1996

$$
q_{t}=c+\alpha q_{t-1}+\sum_{i=1}^{k} \psi_{i} \Delta q_{t-i}+u_{t}
$$

\begin{tabular}{|lccccccc|}
\hline Country & $\mathrm{k}$ & $\alpha_{L S}$ & $95 \% \mathrm{CI}$ & $H L_{\alpha}$ & $95 \% \mathrm{CI}$ & $H L_{\text {IRF }}$ & $95 \% \mathrm{CI}$ \\
\hline Canada & 3 & 0.748 & {$[0.530,0.839]$} & 2.39 & {$[1.09,3.95]$} & 3.27 & {$[1.62,5.14]$} \\
France & 2 & 0.459 & {$[0.202,0.618]$} & 0.89 & {$[0.43,1.44]$} & 1.67 & {$[0.84,2.47]$} \\
Italy & 0 & 0.771 & {$[0.575,0.855]$} & 2.67 & {$[1.25,4.42]$} & 2.67 & {$[1.25,4.42]$} \\
Japan & 4 & 0.940 & {$[0.678,0.975]$} & 11.20 & {$[1.77,27.24]$} & $\infty$ & {$[2.34, \infty)$} \\
Netherlands & 3 & 0.712 & {$[0.494,0.817]$} & 2.04 & {$[0.98,3.43]$} & 3.50 & {$[1.42,4.88]$} \\
UK & 1 & 0.837 & {$[0.656,0.900]$} & 3.90 & {$[1.64,6.58]$} & 4.43 & {$[3.89,4.80]$} \\
\hline
\end{tabular}


Table 3. Exactly Median Unbiased Half-Lives in Dickey-Fuller Regressions Annual Data: 1900-1996

$$
q_{t}=c+\alpha q_{t-1}+u_{t}
$$

\begin{tabular}{|lcccc|}
\hline Country & $\alpha_{M U}$ & $95 \%$ CI & $H L_{M U}$ & $95 \%$ CI \\
\hline Canada & 0.82 & {$[0.69,0.97]$} & 3.49 & {$[1.87,22.76]$} \\
France & 0.62 & {$[0.45,0.79]$} & 1.45 & {$[0.87,2.94]$} \\
Italy & 0.80 & {$[0.66,0.98]$} & 3.11 & {$[1.67,34.31]$} \\
Japan & 0.94 & {$[0.83,1.0]$} & 11.20 & {$[3.72, \infty)$} \\
Netherlands & 0.82 & {$[0.69,0.97]$} & 3.49 & {$[1.87,22.76]$} \\
UK & 0.90 & {$[0.79,1.0]$} & 6.58 & {$[2.94, \infty)$} \\
\hline
\end{tabular}


Table 4. Approximately Median Unbiased Half-Lives in Augmented Dickey-Fuller Regressions. Annual Data: 1900-1996

$$
q_{t}=c+\alpha q_{t-1}+\sum_{i=1}^{k} \psi_{i} \Delta q_{t-i}+u_{t}
$$

\begin{tabular}{|lccccccc|}
\hline Country & $\mathrm{k}$ & $\alpha_{M U}$ & $95 \% \mathrm{CI}$ & $H L_{\alpha, M U}$ & $95 \% \mathrm{CI}$ & $H L_{I R F,}$ & $95 \% \mathrm{CI}$ \\
\hline Canada & 3 & 0.78 & {$[0.59,0.91]$} & 2.79 & {$[1.31,7.35]$} & 4.18 & {$[1.70,7.93]$} \\
France & 2 & 0.47 & {$[0.30,0.66]$} & 0.92 & {$[0.58,1.67]$} & 1.76 & {$[0.86,2.55]$} \\
Italy & 0 & 0.80 & {$[0.66,0.98]$} & 3.11 & {$[1.67,34.31]$} & 3.11 & {$[1.67,34.31]$} \\
Japan & 4 & 1.0 & {$[0.76,1.0]$} & $\infty$ & {$[2.53, \infty)$} & $\infty$ & {$[1.93, \infty)$} \\
Netherlands & 3 & 0.74 & {$[0.54,0.88]$} & 2.30 & {$[1.12,5.42]$} & 3.78 & {$[1.57,6.62]$} \\
UK & 1 & 0.87 & {$[0.72,0.96]$} & 4.98 & {$[2.11,16.98]$} & 5.43 & {$[2.59,17.57]$} \\
\hline
\end{tabular}


Table 5. OLS Half-Lives in Dickey-Fuller Regressions

Quarterly Data: 1973:1-1998:2

$$
q_{t}=c+\alpha q_{t-1}+u_{t}
$$

\begin{tabular}{|lcccc|}
\hline Country & $\alpha_{L S}$ & $95 \%$ CI & $H L_{L S}$ & $95 \%$ CI \\
\hline Australia & 0.951 & {$[0.796,0.977]$} & 3.45 & {$[0.76,7.45]$} \\
Austria & 0.929 & {$[0.764,0.963]$} & 2.35 & {$[0.64,4.60]$} \\
Belgium & 0.946 & {$[0.773,0.973]$} & 3.12 & {$[0.67,6.33]$} \\
Canada & 0.991 & {$[0.866,1.0]$} & 19.17 & {$[1.20, \infty)$} \\
Denmark & 0.935 & {$[0.766,0.967]$} & 2.58 & {$[0.65,5.16]$} \\
Finland & 0.941 & {$[0.773,0.970]$} & 2.85 & {$[0.67,5.69]$} \\
France & 0.932 & {$[0.763,0.964]$} & 2.46 & {$[0.64,4.73]$} \\
Germany & 0.929 & {$[0.761,0.963]$} & 2.35 & {$[0.63,4.60]$} \\
Greece & 0.935 & {$[0.763,0.967]$} & 2.58 & {$[0.64,5.16]$} \\
Ireland & 0.897 & {$[0.716,0.942]$} & 1.59 & {$[0.52,2.90]$} \\
Italy & 0.930 & {$[0.756,0.964]$} & 2.39 & {$[0.62,4.73]$} \\
Japan & 0.955 & {$[0.815,0.980]$} & 3.76 & {$[0.85,8.58]$} \\
Netherlands & 0.925 & {$[0.757,0.959]$} & 2.27 & {$[0.62,4.14]$} \\
New Zealand & 0.926 & {$[0.751,0.960]$} & 2.25 & {$[0.61,4.24]$} \\
Norway & 0.912 & {$[0.743,0.952]$} & 1.88 & {$[0.58,3.52]$} \\
Portugal & 0.957 & {$[0.787,0.978]$} & 3.94 & {$[0.72,7.79]$} \\
Spain & 0.954 & {$[0.791,0.978]$} & 3.68 & {$[0.74,7.79]$} \\
Sweden & 0.948 & {$[0.779,0.974]$} & 3.25 & {$[0.69,6.58]$} \\
Switzerland & 0.864 & {$[0.688,0.920]$} & 1.19 & {$[0.46,2.08]$} \\
United Kingdom & 0.919 & {$[0.753,0.955]$} & 2.05 & {$[0.61,3.76]$} \\
\hline
\end{tabular}


Table 6. OLS Half-Lives in Augmented Dickey-Fuller Regressions Quarterly Data: 1973:1-1998:2

$$
q_{t}=c+\alpha q_{t-1}+\sum_{i=1}^{k} \psi_{i} \Delta q_{t-i}+u_{t}
$$

\begin{tabular}{|lccccccc|}
\hline Country & $\mathrm{K}$ & $\alpha_{L S}$ & $95 \% \mathrm{CI}$ & $H L_{\alpha}$ & $95 \%$ CI & $H L_{\text {IRF }}$ & $95 \% \mathrm{CI}$ \\
& & & & & & & \\
\hline Australia & 3 & 0.930 & {$[0.789,0.963]$} & 2.39 & {$[0.73,4.60]$} & 2.12 & {$[0.97,4.83]$} \\
Austria & 4 & 0.909 & {$[0.762,0.951]$} & 1.82 & {$[0.64,3.45]$} & 2.22 & {$[1.17,4.17]$} \\
Belgium & 4 & 0.921 & {$[0.780,0.956]$} & 2.11 & {$[0.70,3.85]$} & 2.49 & {$[1.26,4.60]$} \\
Canada & 6 & 0.962 & {$[0.861,0.982]$} & 4.47 & {$[1.16,9.54]$} & 4.21 & {$[1.81,10.19]$} \\
Denmark & 3 & 0.923 & {$[0.774,0.959]$} & 2.16 & {$[0.68,4.14]$} & 2.07 & {$[1.07,4.59]$} \\
Finland & 7 & 0.891 & {$[0.756,0.937]$} & 1.50 & {$[0.62,2.66]$} & 2.36 & {$[1.19,3.84]$} \\
France & 4 & 0.901 & {$[0.749,0.945]$} & 1.66 & {$[0.59,3.06]$} & 2.12 & {$[1.14,3.71]$} \\
Germany & 4 & 0.904 & {$[0.763,0.947]$} & 1.72 & {$[0.64,3.18]$} & 2.16 & {$[1.17,3.90]$} \\
Greece & 4 & 0.918 & {$[0.759,0.956]$} & 2.03 & {$[0.63,3.85]$} & 2.35 & {$[1.18,4.69]$} \\
Ireland & 7 & 0.890 & {$[0.666,0.942]$} & 1.49 & {$[0.43,2.90]$} & 1.36 & {$[0.49,3.18]$} \\
Italy & 4 & 0.897 & {$[0.736,0.942]$} & 1.59 & {$[0.57,2.90]$} & 1.98 & {$[1.04,3.53]$} \\
Japan & 3 & 0.944 & {$[0.817,0.972]$} & 3.01 & {$[0.86,6.10]$} & 2.52 & {$[1.17,6.37]$} \\
Netherlands & 4 & 0.897 & {$[0.746,0.942]$} & 1.59 & {$[0.59,2.88]$} & 2.14 & {$[1.17,3.62]$} \\
New Zealand & 3 & 0.884 & {$[0.751,0.930]$} & 1.41 & {$[0.61,2.39]$} & 1.85 & {$[1.08,2.97]$} \\
Norway & 7 & 0.887 & {$[0.684,0.938]$} & 2.08 & {$[0.46,2.71]$} & 2.14 & {$[0.78,3.85]$} \\
Portugal & 8 & 0.937 & {$[0.778,0.971]$} & 2.66 & {$[0.69,5.89]$} & 3.15 & {$[1.16,6.88]$} \\
Spain & 8 & 0.909 & {$[0.762,0.951]$} & 1.82 & {$[0.64,3.45]$} & 2.97 & {$[1.14,4.91]$} \\
Sweden & 8 & 0.896 & {$[0.762,0.941]$} & 1.58 & {$[0.64,2.85]$} & 2.87 & {$[1.08,4.61]$} \\
Switzerland & 4 & 0.869 & {$[0.661,0.927]$} & 1.23 & {$[0.42,2.29]$} & 1.24 & {$[0.22,2.68]$} \\
United Kingdom & 7 & 0.868 & {$[0.702,0.925]$} & 1.22 & {$[0.49,2.22]$} & 2.13 & {$[0.80,3.20]$} \\
\hline
\end{tabular}


Table 7. Exactly Median Unbiased Half-Lives in Dickey-Fuller Regressions Quarterly Data: 1973:1-1998:2

$$
q_{t}=c+\alpha q_{t-1}+u_{t}
$$

\begin{tabular}{|lcccc|}
\hline Country & $\alpha_{M U}$ & $95 \%$ CI & $H L_{M U}$ & $95 \%$ CI \\
\hline Australia & 0.99 & {$[0.91,1.0]$} & 17.24 & {$[1.84, \infty)$} \\
Austria & 0.97 & {$[0.86,1.0]$} & 5.69 & {$[1.15, \infty)$} \\
Belgium & 0.99 & {$[0.90,1.0]$} & 17.24 & {$[1.64, \infty)$} \\
Canada & 1.0 & {$[0.95,1.0]$} & $\infty$ & {$[8.58, \infty)$} \\
Denmark & 0.98 & {$[0.89,1.0]$} & 8.58 & {$[1.49, \infty)$} \\
Finland & 0.98 & {$[0.90,1.0]$} & 8.58 & {$[1.64, \infty)$} \\
France & 0.97 & {$[0.88,1.0]$} & 5.69 & {$[1.36, \infty)$} \\
Germany & 0.97 & {$[0.88,1.0]$} & 5.69 & {$[1.36, \infty)$} \\
Greece & 0.98 & {$[0.89,1.0]$} & 8.58 & {$[1.49, \infty)$} \\
Ireland & 0.93 & {$[0.83,1.0]$} & 2.39 & {$[0.93, \infty)$} \\
Italy & 0.97 & {$[0.88,1.0]$} & 5.69 & {$[1.36, \infty)$} \\
Japan & 1.0 & {$[0.92,1.0]$} & $\infty$ & {$[2.08, \infty)$} \\
Netherlands & 0.96 & {$[0.87,1.0]$} & 4.24 & {$[1.24, \infty)$} \\
New Zealand & 0.96 & {$[0.87,1.0]$} & 4.24 & {$[1.24, \infty)$} \\
Norway & 0.95 & {$[0.85,1.0]$} & 3.38 & {$[1.07, \infty)$} \\
Portugal & 1.0 & {$[0.92,1.0]$} & $\infty$ & {$[2.08, \infty)$} \\
Spain & 1.0 & {$[0.92,1.0]$} & $\infty$ & {$[2.08, \infty)$} \\
Sweden & 0.99 & {$[0.91,1.0]$} & 17.24 & {$[1.84, \infty)$} \\
Switzerland & 0.90 & {$[0.79,1.0]$} & 1.64 & {$[0.74, \infty)$} \\
United Kingdom & 0.96 & {$[0.87,1.0]$} & 4.24 & {$[1.24, \infty)$} \\
\hline
\end{tabular}


Table 8. Approximately Median Unbiased Half-Lives in Augmented Dickey-Fuller Regressions. Quarterly Data: 1973:1-1998:2

$$
q_{t}=c+\alpha q_{t-1}+\sum_{i=1}^{k} \psi_{i} \Delta q_{t-i}+u_{t}
$$

\begin{tabular}{|lccccccc|}
\hline Country & $\mathrm{k}$ & $\alpha_{M U}$ & $95 \% \mathrm{CI}$ & $H L_{\alpha, M U}$ & $95 \% \mathrm{CI}$ & $H L_{I R F, M}$ & $95 \% \mathrm{CI}$ \\
& & & & & & & \\
\hline Australia & 3 & 0.97 & {$[0.83,1.0]$} & 5.69 & {$[0.93, \infty)$} & 6.13 & {$[1.12, \infty)$} \\
Austria & 4 & 0.93 & {$[0.80,1.0]$} & 2.39 & {$[0.78, \infty)$} & 3.10 & {$[1.24, \infty)$} \\
Belgium & 4 & 0.94 & {$[0.82,1.0]$} & 2.80 & {$[0.87, \infty)$} & 3.57 & {$[1.39, \infty)$} \\
Canada & 6 & 0.99 & {$[0.87,1.0]$} & 17.24 & {$[1.24, \infty)$} & 17.70 & {$[1.99, \infty)$} \\
Denmark & 3 & 0.95 & {$[0.81,1.0]$} & 3.38 & {$[0.82, \infty)$} & 3.74 & {$[1.18, \infty)$} \\
Finland & 7 & 0.91 & {$[0.78,0.98]$} & 1.84 & {$[0.70,8.58]$} & 2.97 & {$[1.25,11.19]$} \\
France & 4 & 0.92 & {$[0.78,1.0]$} & 2.08 & {$[0.70, \infty)$} & 2.80 & {$[1.23, \infty)$} \\
Germany & 4 & 0.93 & {$[0.80,1.0]$} & 2.39 & {$[0.78, \infty)$} & 3.03 & {$[1.24, \infty)$} \\
Greece & 4 & 0.94 & {$[0.79,1.0]$} & 2.80 & {$[0.74, \infty)$} & 3.52 & {$[1.27, \infty)$} \\
Ireland & 7 & 0.93 & {$[0.75,1.0]$} & 2.39 & {$[0.60, \infty)$} & 2.77 & {$[1.00, \infty)$} \\
Italy & 4 & 0.92 & {$[0.78,1.0]$} & 2.08 & {$[0.70, \infty)$} & 2.78 & {$[1.24, \infty)$} \\
Japan & 3 & 0.97 & {$[0.84,1.0]$} & 5.69 & {$[0.99, \infty)$} & 6.12 & {$[1.37, \infty)$} \\
Netherlands & 4 & 0.92 & {$[0.78,0.99]$} & 2.08 & {$[0.70,17.24]$} & 2.98 & {$[1.24,18.59]$} \\
New Zealand & 3 & 0.91 & {$[0.79,0.98]$} & 1.84 & {$[0.74,8.58]$} & 2.49 & {$[1.25,9.26]$} \\
Norway & 7 & 0.92 & {$[0.76,1.0]$} & 2.08 & {$[0.63, \infty)$} & 3.03 & {$[0.98, \infty)$} \\
Portugal & 8 & 0.97 & {$[0.83,1.0]$} & 5.69 & {$[0.93, \infty)$} & 6.54 & {$[1.20, \infty)$} \\
Spain & 8 & 0.93 & {$[0.79,1.0]$} & 2.39 & {$[0.74, \infty)$} & 3.78 & {$[1.22, \infty)$} \\
Sweden & 8 & 0.91 & {$[0.78,1.0]$} & 1.84 & {$[0.70, \infty)$} & 3.22 & {$[1.11, \infty)$} \\
Switzerland & 4 & 0.91 & {$[0.75,1.0]$} & 1.84 & {$[0.60, \infty)$} & 2.20 & {$[0.23, \infty)$} \\
United Kingdom & 7 & 0.90 & {$[0.73,0.99]$} & 1.64 & {$[0.55,17.24]$} & 2.71 & {$[0.92,18.52]$} \\
\hline
\end{tabular}

